\title{
PARALLEL INPUTS TO MEMORY IN BEE COLOUR VISION*
}

\author{
ADRIAN HORRIDGE** \\ Australian National University, Canberra, ACT 0200, Australia
}

(Received: August 28, 2015; accepted: September 18, 2015)

\begin{abstract}
In the $19^{\text {th }}$ century, it was found that attraction of bees to light was controlled by light intensity irrespective of colour, and a few critical entomologists inferred that vision of bees foraging on flowers was unlike human colour vision. Therefore, quite justly, Professor Carl von Hess concluded in his book on the Comparative Physiology of Vision (1912) that bees do not distinguish colours in the way that humans enjoy. Immediately, Karl von Frisch, an assistant in the Zoology Department of the same University of Münich, set to work to show that indeed bees have colour vision like humans, thereby initiating a new research tradition, and setting off a decade of controversy that ended only at the death of Hess in 1923. Until 1939, several researchers continued the tradition of trying to untangle the mechanism of bee vision by repeatedly testing trained bees, but made little progress, partly because von Frisch and his legacy dominated the scene. The theory of trichromatic colour vision further developed after three types of receptors sensitive to green, blue, and ultraviolet (UV), were demonstrated in 1964 in the bee. Then, until the end of the century, all data was interpreted in terms of trichromatic colour space. Anomalies were nothing new, but eventually after 1996 they led to the discovery that bees have a previously unknown type of colour vision based on a monochromatic measure and distribution of blue and measures of modulation in green and blue receptor pathways. Meanwhile, in the $20^{\text {th }}$ century, search for a suitable rationalization, and explorations of sterile culs-de-sac had filled the literature of bee colour vision, but were based on the wrong theory.
\end{abstract}

Keywords: Honeybee - insect colour vision - von Hess - von Frisch - contrast - modulation - monochromatic blue - new beginning

\section{INTRODUCTION}

Adherence to tradition is a great virtue most of the time. Tradition saves thought and effort of discovering how to proceed; it short-circuits worry, allows everyone to predict the behaviour of the neighbours and generates confidence in the validity of knowledge. I support tradition, but scientific research is the implacable enemy of tradition. New facts destroy old ideas so that traditional ways often shift painfully to Hungary.

*Presented at the ISIN Symposium on Invertebrate Neurobiology, 26-30 August, 2015, Tihany,

**E-mail address: horridge@netspeed.com.au 
new paths. My grandfather was owner of a factory in Sheffield, England, that made fine goods from ivory, ebony, animal horns, antlers, amber, turtle shell, and mother of pearl. Vikings in the $7^{\text {th }}$ century brought this traditional craft across the North Sea and up the River Don. It ended in 1922 when the land and factory were sold because newly invented plastics replaced animal products.

In science, major upsets are fortunately rare and never so sharp, because science is never just one theory or one person's property. Science requires varied activities of many experts, trained in different places, creating change at different speeds. Usually the actual change is disgracefully slow because tradition and belief have immensely strong drag.

Our story to be told began when men first kept bees, and presumed from their own behaviour that bees also distinguish colours of flowers where they forage. Karl von Frisch [4] accepted this reasonable presumption before he began with a standard test for faults in human colour vision. He successfully trained bees to distinguish coloured papers (except grey and blue-green) from a selection of grey ones. When Carl von Hess [6] trained bees on a blue/yellow checkerboard, those trained on blue would later prefer blue but those changed on yellow squares had no particular colour preference. Neither understood their own results, and the contradiction was never resolved. After the death of Hess in 1923, colour vision was accepted despite the anomalous results of one and contradictory results of the other. Much later, Maxwell's theory of trichromatic colour space (developed for humans) was greatly strengthened for bees by the measured spectral sensitivity of three types of photoreceptors with peaks in the blue, green, and UV parts of the spectrum [1]. In each ommatidium there are six green sensitive ending at the lamina, one blue and one UV ending in the medulla [21]. Further colour vision anomalies that appeared at the end of the $20^{\text {th }}$ century suggested that bees detect contrast at edges, not a variety of colours [11,12]. Recent discoveries show that bees see neither colours nor shapes of flowers, but they use a newly identified mechanism with just three independent inputs behind each facet of the compound eye. These inputs are the tonic input of blue and the phasic inputs from green and blue contrast $[13,14,16]$. The automatic measurement of angles between these inputs is a consequence of compound eye structure [14]. Ultraviolet (UV) might make a fourth input, but was excluded from the apparatus in the experiments that follow. This exclusion probably does not matter because UV inhibits the blue channel in bees [10] and UV has never been seriously implicated in bee colour vision. The experiments that follow illustrate the action of primary inputs into the bee visual memory in the foraging behaviour.

\section{METHODS}

The experiments were done in the Y-choice apparatus (Fig. 1), which has been described many times $[11,12]$. The baffles of transparent Perspex $1 \mathrm{~mm}$ thick forced incoming bees to stop and look at two targets that subtended an angle of about $55^{\circ}$ from the point of choice. The walls of the apparatus were white; the top was of clear 


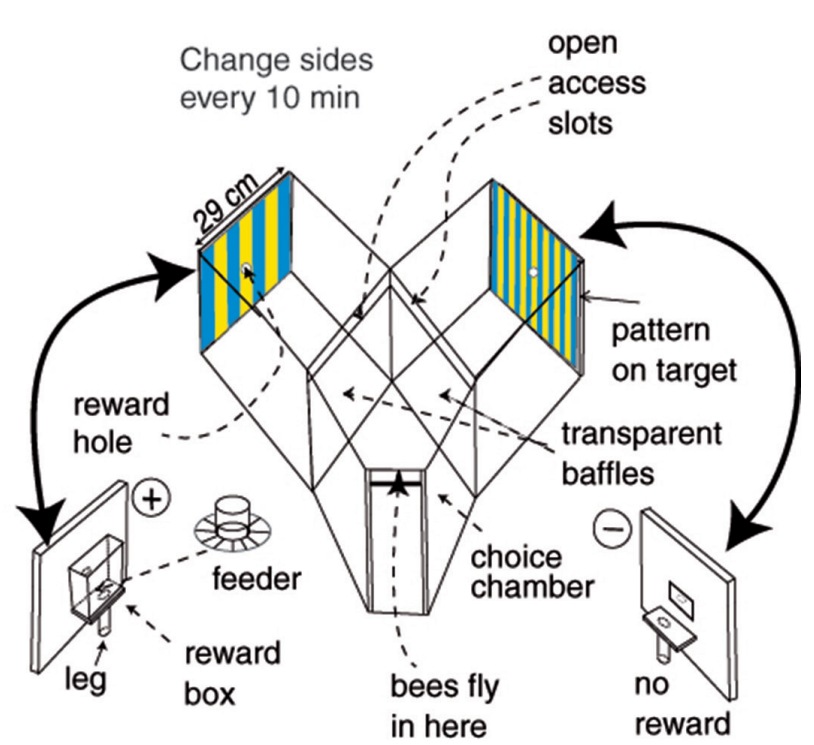

Fig. 1. The Y-choice apparatus. The bees entered through a narrow horizontal slot at top front into a choice chamber from which they could see both targets. They selected one side, passed over one of the transparent baffles through the narrow horizontal slot and reached the reward hole. When satisfied, they exited by the way they came. To make the bees look at the patterns and learn something, the reward with its pattern changed sides every 5 or $10 \mathrm{~min}$

Perspex that absorbed UV. From their point of decision behind the baffles, bees could resolve the reward hole and vertical edges of each target. The reward was a fresh aqueous solution of sucrose sufficiently concentrated to keep the marked bees making regular visits without attracting recruits. During the training and tests the side of the positive target and of the reward with it were changed every $5 \mathrm{~min}$ to prevent the bees from learning which arm of the apparatus to choose, but in the illustrations the rewarded pattern (labelled + ) was on the left.

The targets carried patterns made with papers from the standard series manufactured by Canson (France), and available in artist's stores. To avoid the profusion of names in the literature, colour names are those published by the manufacturer. Calibrations of stimuli displayed by each paper to blue and green receptors, relative to plain white copying paper, are given in Table 1.

A small group of 10-15 individually marked bees from a local hive was trained to select one of the two targets while in flight. The criterion for a score was when a bee passed one baffle or the other. First choice of each identified bee in each period of 5 min was recorded while training continued. These results were labelled "train". To obtain the results labelled "test", a different pair of patterns was inserted and choices were recorded individually for $5 \mathrm{~min}$ on each side. All tests were made with one pattern rewarded, and then at a different time with the other pattern rewarded. In tests it was essential to give a reward, otherwise the bees continued to search. All tests were repeated with the patterns reversed in the two arms of the apparatus to compensate 


\begin{tabular}{|l|c|c|}
\multicolumn{1}{|c|}{ Canson papers } & Blue receptor & Green receptor \\
\hline White copy paper & 100 & 100 \\
\hline Hemp 374 & 34.2 & 56.3 \\
\hline Ultramarine 590 & 33.8 & 20.7 \\
\hline Green 576 & 17.0 & 22.3 \\
\hline Buff 384 & 25.7 & 41.7 \\
\hline Blue 595 & 54.2 & 40.0 \\
\hline Contrast 374/590 & 0.006 & 0.46 \\
\hline Contrast 384/595 & 0.36 & 0.02 \\
\hline
\end{tabular}

Relative receptor stimuli from the different papers relative to the white paper (100\%), and contrasts between two equiluminant pairs of papers. White displays by far the greatest stimulus because it spreads maximum emission over the whole wavelength range of each receptor type. Colour names and numbers are of the manufacturer, Canson. See: http://www.canson-infinity.com/en/values.asp

for possible side preferences. Several different tests were interleaved during continued periods of training, so the trained bees did not become familiar with any one test.

Because the results were frequencies, an estimate of the s.d. was the value of $\sqrt{ }[\mathrm{p}(1-\mathrm{p}) / \mathrm{n}]$ where $\mathrm{p}$ was the fraction of correct choices and $\mathrm{n}$ was the total number of choices. This method assumed that there were no trends during counting, that the individual choices were independent and they had a binomial distribution about the mean. A score of $57 \%$ based on 200 choices, or $60 \%$ based on 100 choices, was twice the estimated standard deviation away from the null (random) hypothesis of $50 \%$. If the s.d. was used, $\mathrm{p}<0.05$ for a score of $57 \%, \mathrm{p}<0.01$ for a score of $60 \%$ for 200 choices. If the chi-squared test was used, $\mathrm{p}<0.05$ for a score of $62 \%, \mathrm{n}=100, \mathrm{p}<0.02$ for a score of $62 \%$, and $\mathrm{p}<0.002$ for a score of $65 \%$.

Quantitative comparisons of the scores in different tests are not as valid as might be thought, because each test is a forced choice, therefore should be $100 \%$ if the bees have learned, and $50 \%$ if they have not. Each test involves a different mixture of several cues for which the bees have an order of preference. The bees switch between cues as the tests require, so it is hard to justify numerical comparisons of scores in different tests. The main requirement was a decision as to whether bees could or could not discriminate in each test, from which a little logic revealed the cue they had used. To do this, it was essential to reduce the number of variables by use of pairs of coloured papers that were equiluminant to blue or to green receptor channels, and to design patterns that displayed few cues. Mediocre scores hide the true state of bee education. A score of $60 \%$ correct is compatible with $80 \%$ of choices made at random. It was far better to use subtle training patterns that gave high scores, and design conclusive tests, than to struggle with marginally significant test results. 
Bees find their own preferred cues, not what you would intuitively expect

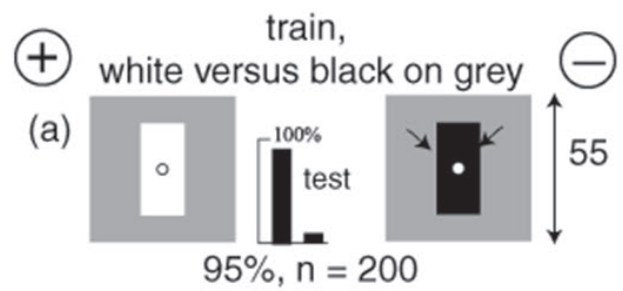

(b)

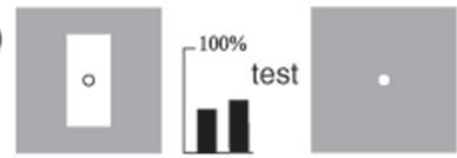

$47 \%, n=100$

They had not learned white

(c)

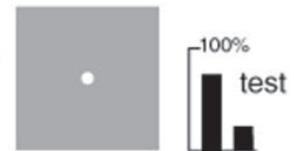

$81 \%, n=100$

They learned unrewarded target

(d)

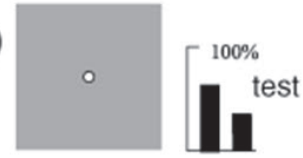

$62 \%, n=100$

They avoided black bars

(e)

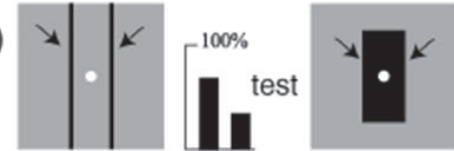

$71 \%, n=100$

They avoided edges of black more than black bars (f)

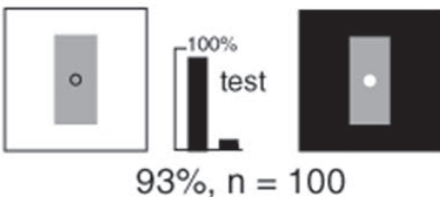

They accepted contrast reversal

(g)

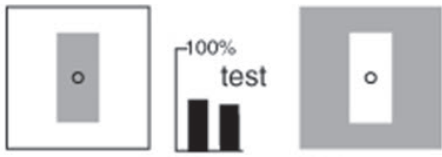

$51 \%, n=100$

Polarity of contrast was ignored

(h)

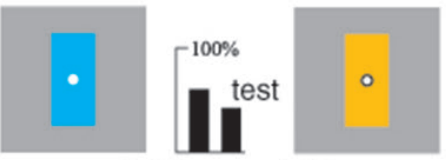

$64 \%, n=100$

With equal green contrast, they avoided least blue

(j)

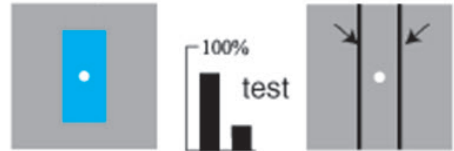

$78 \%, n=100$

Avoided bars and preferred blue

\section{Conclusion: They learned position of greatest green modulation on the unrewarded target and avoided least blue target.}

Fig. 2. Contrast was not the stimulus. (a) Bees were trained on white versus black, both on a grey background of 50\% white. (b and c) The trained bees recognized only the unrewarded target and avoided it. (d and e) They avoided vertical edges with the strongest green contrast. (f) Reversal of contrast on both targets had no effect on good recognition. (g) Equal contrast of opposite polarity was useless as a cue ( $h$ and $j$ ) With equal green contrast, the trained bees preferred blue learned from the white. (j) They avoided vertical bars and preferred blue 


\section{RESULTS}

\section{Bees detect edges, but not which side is which. Edge polarity is lost}

Bees quickly learned to distinguish a white rectangle from a black one of equal size, each on a grey background of 50\% white (Fig. 2a). Lengths of edge and widths between edges were identical, so offered no cue. The contrast between white and grey was the same but in the opposite direction as the contrast between black and grey. The bees learned to avoid the unrewarded target but not the rewarded one (Fig. 2b and c), Initial training was not classical conditioning. They searched actively, learned by trial and error, and remembered their errors. The next two tests showed that they learned to avoid thin black bars representing edges of the unrewarded black rectangle, and to avoid even more the edges of the black rectangle (Fig. $2 \mathrm{~d}$ and e), which was the strongest concentration of the preferred stimulus [16].

Next, the trained bees distinguished between grey on white versus grey on black as accurately as in the training (Fig. 2f) as if they were insensitive to the polarity of the contrast, something that humans would never do. However, they confused white on grey with grey on white, although the size and polarity of the contrasts were the same as in the training targets (Fig. 2 g). Clearly, the features that humans use, actual contrasts of grey, black and white, or polarity at edges, were not the cue. A test with blue versus buff, which were equiluminant for the green receptors, revealed an attraction for the greater blue content (Fig. 2h), and we know from other work [16] that the preferred input was the strongest green contrast, indicated here by arrows (Fig. 2a, d, e and $\mathrm{j}$ ). In the training, the bees were not interested in the actual contrast or its polarity at boundaries.

\section{Contrast alone is an incomplete variable in insect vision}

Contrast is the change in stimulus detected as a photoreceptor scans across a boundary or an edge against background. It is a principal variable in human psychophysics because the contrast defines edges of objects and areas that we recognize and categorize. Human vision cannot avoid seeing contrast; it is impossible not to see the polarity at an edge, and the panorama is inconceivable without directional steps between areas of colour, black or white. In studies of insect vision, responses of large $2^{\text {nd }}$ order neurons of the lamina are optimized to detect contrast [17]. Bees certainly detect and locate edges but not their polarity, but they do not distinguish which side of the edge is which, as was discovered but not understood long ago [5]. This is difficult to imagine until we realize that all symmetrical detectors behave so. A detector that scans an edge cannot detect the polarity when the response is the same for each direction of scanning, although the edge position is located and learned. Bee edge detectors certainly act as if they are symmetrical, e.g. white/grey = black/grey (Fig. 2f) or grey/ white = white/grey (Fig. 2g).

Our first awkward informative fact about insect vision is that the layout of a coloured pattern cannot be reconstructed because much of the information about spatial 
distribution of contrast in the image is thrown away right at the start to reduce the information load. Contrast at vertical edges is certainly detected and measured as modulation, but informative studies of the part played by contrast in the bees' feeding behaviour are hard to find for a different reason; the relevant variable was thought to be colour. Adding to the essential reduction of information, the green receptor pathway in bees is not sensitive to differences in brightness (except near threshold).

\section{Green and blue channels separated by EQUILUMINANCE}

Train, equiluminant to green, and no colour difference

(a)

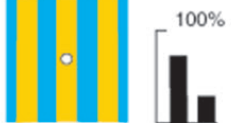

$75.0 \%, n=200$

(b)

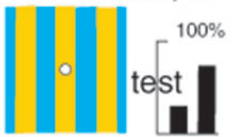

$34.0 \%, n=200$ Trained bees
Blue contrast

(c)

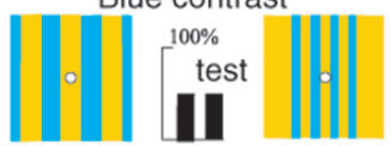

$49.5 \%, n=200$

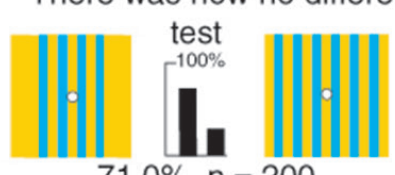

$71.0 \%, n=200$ (e)

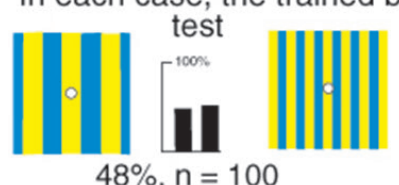

Green contrast
Train, equiluminant to blue and no colour difference

(f)

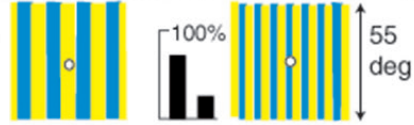

$76.5 \%, n=200$

(g)

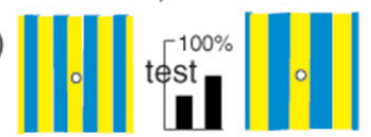

$41.0 \%, n=200$

odulation

(h)

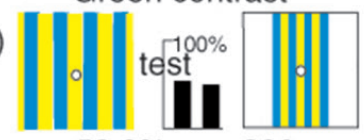

$53.0 \%, n=200$

.




\section{Recognition of patterns of edges by a measure of total modulation, not contrast}

Bees very easily distinguish between two targets of parallel vertical gratings with differing period, that is, different total lengths of vertical edge (Fig. 3a, f). Such gratings are equal in area of each colour, therefore equal in total colour, which is a significant variable because the individual bars cannot be visualized separately without a large brain. Colours of grating bars are smoothed together in the blue receptor channel for colour detection, but the green receptor channel measures the total of amount of (vertical bar edge $\times$ contrast) as modulation.

After training to avoid the pattern with more edge, with no green contrast (Fig. 3a), bees were attracted to a pattern with even less edge, and abandoned the rewarded target in the training (Fig. 3b). They had learned the relative, not absolute, measure of edge with blue contrast. When tested with an equal number of bars on each target (Fig. 3c), the trained bees were lost, although the period on each target was identical to that in the training. Therefore, they had learned a measure of modulation from the total length of vertical edge on each target, not the widths of the patterns. It must have been blue receptor modulation because there was no green contrast in the training targets. Using the same cue, the trained bees easily distinguished between targets with the same period but different numbers of bars (Fig. 3d), but were lost with gratings that displayed green contrast and equiluminant to blue receptors (Fig. 3e).

When trained on similar gratings that were equiluminant to the blue receptors (Fig. 3f), the same tests gave similar results (Fig. $3 g-j$ ) because the properties of green and blue contrast are similar, but this time the trained bees were unable to distinguish gratings with no green contrast (Fig. 3k).

\section{The informative variable and first preference is a measure of modulation}

Actually, it was von Frisch [4] who discovered that flower-like patterns with many petals were easily discriminated from those of equal size with few petals. The topic was thoroughly explored by Zerrahn [22] and Hertz [8], who found in training experiments that shapes of similar size are more easily distinguished the more they differed in total length of edge. Hertz called this variable the "figural intensity" and defined it as the total length of edge, without saying over what area or angle the measurements were made, but already aware that it was not related to distinguishing colour or position. It was inferred that more edge simply generated more flicker as the eye moved.

Contrast and length of edge are inseparably combined into modulation of receptors as bees in flight scan in the horizontal plane. Until we know better, modulation is defined as (total length of vertical edge) multiplied by (contrast at each part of the edge) and is separate in green and blue receptor pathways.

At each point right down to the finest resolution of our eyes, human vision manages to separate the qualities of the light from the geometry of the pattern, and then go further and compensate for chromatic aberrations of the lens. Bees learn the posi- 
tion and measure of total modulation but discard the lay-out of pattern right from the start of processing. They also locate and measure the strongest concentration of green modulation on the target [16].

Our second awkward informative fact about insect vision is that colour and pattern are mostly thrown away, but measures of contrast and edge length are bound together, measured as one, and learned. This is a logical deduction from the results of testing (Fig. 3). Such an anti-intuitive mechanism was not considered until recently [13-16].

\section{White square on equal area of black versus a black square on equal area of white}

Bees also easily distinguished between a white square on a black background from a black square of equal size on a white background (Fig. 4a). Black and white areas were equal on each pattern and there was no average colour difference, therefore total

\section{Train with equal colour and equal areas of black and white}

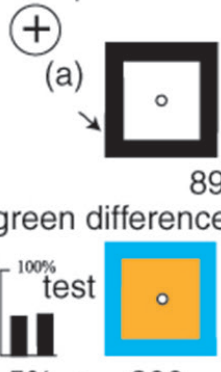

Failed, $48.5 \%, \mathrm{n}=200$

Test with no blue difference

(c)

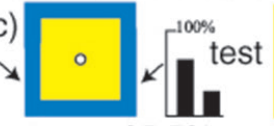

$65.5 \%, n=200$

Test with no green difference train

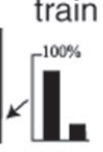

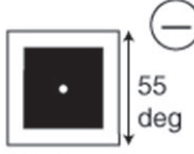

deg

$89.0 \%, n=200$

(d)

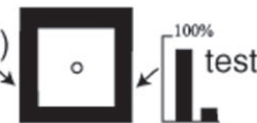

$84.5 \%, \mathrm{n}=200$

(e)

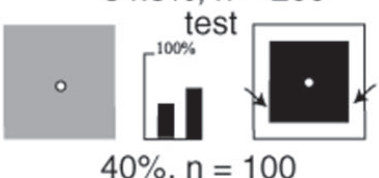

$40 \%, n=100$

They had learned the strongest green modulation at the edges

of the rewarded target, but little from the unrewarded one

(f)

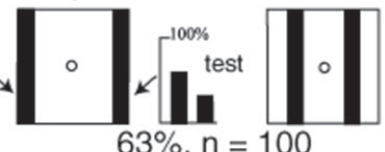

They preferred the greater width

Conclusion: With no colour difference between the targets, they learned the position of the strongest green modulation.

Fig. 4. Bees use colour with black/white patterns (a) Bees were trained with white on an equal area of black, versus black on equal area of white. (b) When tests were on buff and blue, equiluminant to green, they failed. (c) With ultramarine and hemp, equiluminant to blue, they preferred the strong modulation at

the outer vertical edges. (d, e and f) They preferred the target with vertical edges fully separated 
blue content was not a possible cue. Because there was equal blue content on the two targets (Fig. 4a), and because bees have no receptors for white or black, the trained bees failed in a test with similar patterns that were equiluminant to the green receptors (Fig. 4a). Green channel modulation was essential, unlike the previous experiment (Fig. 2h).

The trained bees distinguished targets that were equiluminant for the blue channel because the green channel detected a familiar input (Fig. 4b). Tests with each training target versus a grey $(50 \%$ white) target (Figs $4 \mathrm{~d}$ and $4 \mathrm{e})$ showed that green contrast on both targets had been learned (arrows). A final test showed that the trained bees preferred bars that were most widely separated, showing that they had learned the location of the strongest green modulation and measured the width (arrows). They used an unexpected cue because the training patterns had equal blue content and identical modulation at edges.

When bees distinguished between two black/white patterns, the first preference was to locate and measure the strongest green contrast, which was at vertical edges of black, and the width or angles subtended between vertical edges. Therefore that memory was mainly structural, that is, dependent on what the geometry offered. Also, the locations and relative amounts of blue were learned only where they differed (Figs $2 \mathrm{a}$ and $2 \mathrm{~h}$, not Figs $4 \mathrm{a}$ and $4 \mathrm{~b}$ ).

Humans obviously do not see colour in black/white patterns, but bees measure regional blue content everywhere except in the black (Fig. 2h). All cues, including blue content are ignored when equal on the two targets (Figs 3 and 4) because what is learned on one is unlearned on the other. The trained bees were obliged to learn the width between vertical edges (Fig. 3f) because other preferred cues were not available. One would expect that all patterns of this type with equal areas of two colours (for example, green on white versus white on green) would be distinguished by position of green contrast irrespective of the colours.

\section{Sensitive measurement of blue content}

A blue square on a grey background of $40 \%$ white was discriminated from a plain grey target, also $40 \%$ white (Fig. 5a). The rewarded target had more blue content because the blue paper displayed the blue equivalent of $54.2 \%$ white but displaced the same grey area of $40 \%$ white. A test with the same area of blue on each target, but with different length of edges that displayed some green and blue modulation (Fig. 5b) showed that a modulation difference had also been learned in the training.

When the rewarded target was tested versus plain grey of 50\% white, there was sufficient additional blue to reduce the score (Fig. 5c), but plain grey of $60 \%$ white, made the bees reverse preference (Fig. 5d). Similarly, with 50\% white on each background, the blue square was preferred (Fig. 5e). With $60 \%$ white on each background, the preference was again reversed (Fig. 5f) because the square was less blue than background grey. Despite the lack of blue, a buff square on $40 \%$ white (with similar 
Total blue content was measured over the whole target Numbers in bold give the $\%$ content of white in the grey

Train with blue content

( and low green contrast
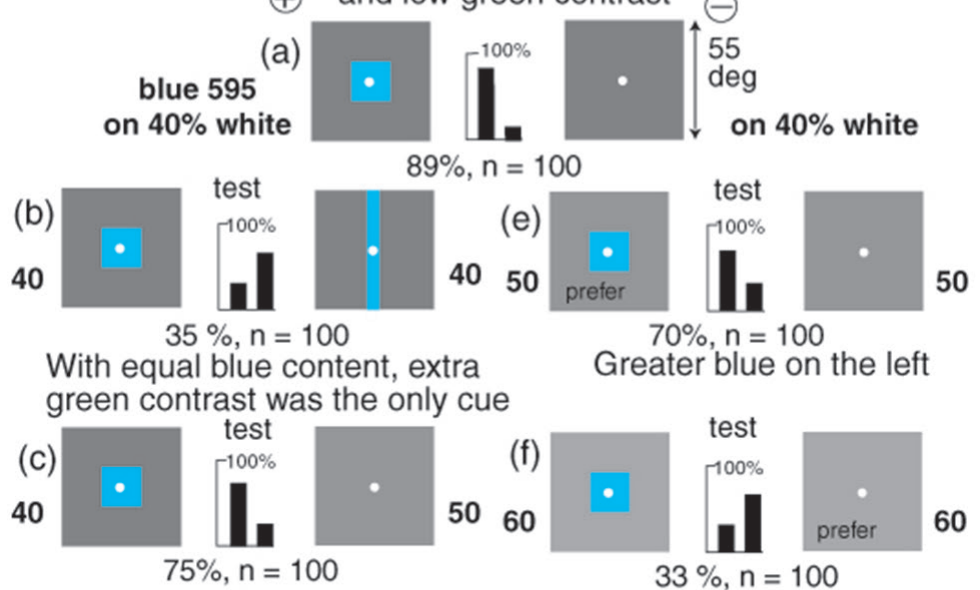

Greater blue on the left
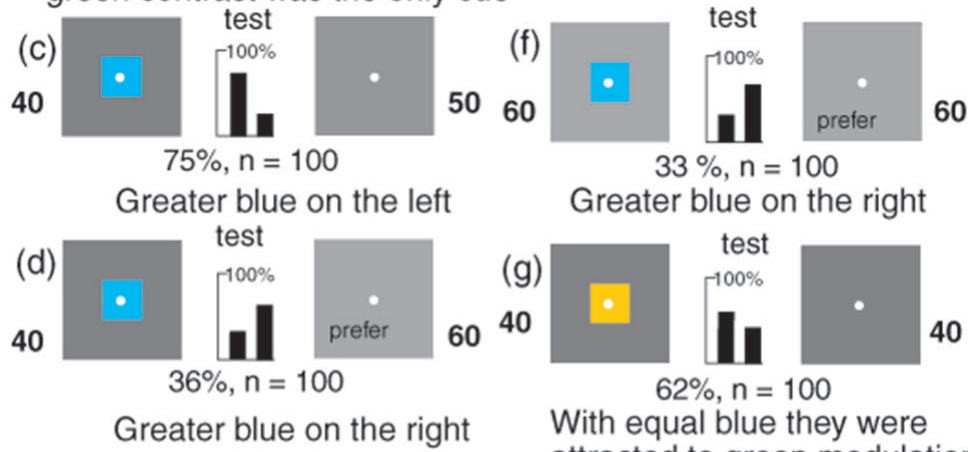

$33 \%, n=100$

Greater blue on the right

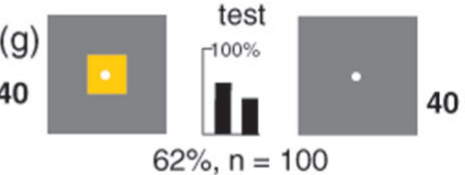

With equal blue they were attracted to green modulation

The square was preferred if its target displayed more blue than the other target, but the square itself was unchanged. Cue was total blue content because one target was blank.

Fig. 5. Blue content is totalled over the whole target. (a) Bees were trained with two grey targets of $40 \%$ white, one with a blue square that displayed $54.2 \%$ white and obscured some grey. (b) With equal areas of blue on $40 \%$ white, they preferred the target with most vertical edges. (c to f) In tests with grey backgrounds of selected content of white, the trained bees preferred the target that displayed most blue content summed over the whole target. (g) When blue content was equal, they preferred green modulation. Numbers in bold show the percentage of white in the grey background

green contrast) was preferred to plain $40 \%$ white, because some blue or green modulation had been learned.

These results show that the blue content was measured over the whole target and the content of blue in the grey background was just as effective as the blue displayed in the blue paper. Our third informative fact is that the field size must have been large because blue content in the square was summed along with the rest of the target, not separated from background. In the training, the bees were obliged to sum blue over 
the whole targets because one target was blank with no pattern at all. The next experiment, however, shows that bees can also measure and learn local blue content when required to do so.

\section{The location of a square was learned from the position of a lack of blue content and some blue contrast.}

Train with equal blue content

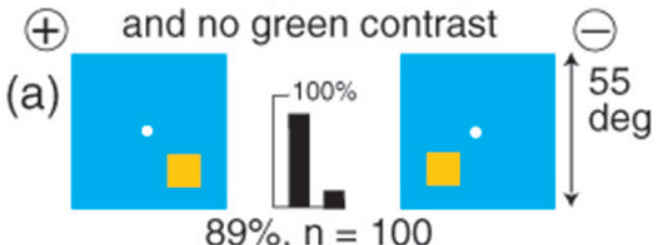

Position of blue and blue modulation were the only cues

(b)

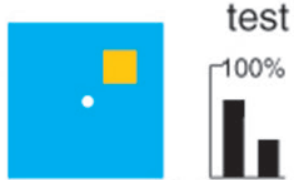

$65 \%, n=100$

Not sensitive to vertical shift, so the detector had a large field

(c)

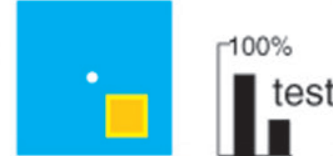

$72 \%, n=100$

Green modulation had little effect, because blue was widespread

(d)

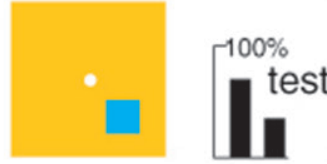

$60 \%, n=100$

Low score with reversal of contrast, because bees used blue modulation (e)

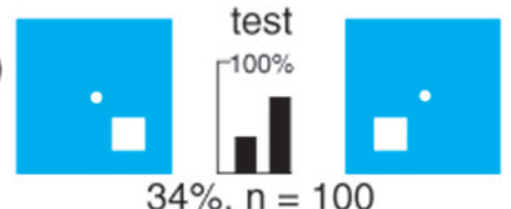

White was bluer than background causing reversal of preference

(f)

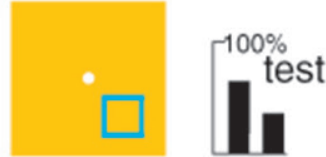

$67 \%, n=100$

Blue modulation was detected irrespective of contrast reversal

(g)
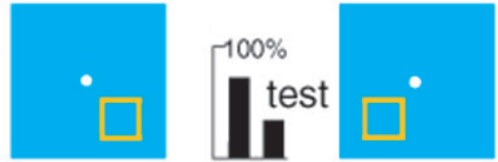

$69 \%, n=100$

Blue modulation was detected

Fig. 6. Position of blue contrast and a local lack of blue was learned. (a) Bees were trained to locate a buff square on blue, with no green contrast. There was a lack of blue where the square obscured the background. (b and c) Moving the squares up or adding green contrast had little effect. (d) Reversal of contrast had little effect so bees had located blue contrast. (e) White squares reversed the preference because white was more blue than blue (see Table 1). ( $\mathrm{f}$ and $\mathrm{g}$ ) Position of blue modulation had been learned 


\section{A small coloured patch was located by the position of displaced blue}

After a brief training, a small buff square at the bottom right corner of the rewarded target on a blue background was distinguished from a similar square at the bottom left corner of the unrewarded target (Fig. 6a). There was no green contrast (Table 1). The squares could be moved upwards on their targets and still be detected; therefore the fields of the detectors must be large (Fig. 6b). Adding a narrow yellow margin to each square reduced the score (Fig. 6c) because green contrast inhibited blue contrast. Some recognition persisted even after reversal of the training colours (Fig. 6d), showing that blue contrast had also been learned.

Replacement of buff by white reversed the preference because buff reduced the local blue content but white increased it. Therefore, the centre of gravity of blue content was shifted to the opposite side of the target. This anti-intuitive behaviour is merely the mechanism at work, but must have consequences for the attractions of flower colours. Positions of hollow blue squares on a buff background, and hollow buff squares on a blue background, were distinguished (Fig. 6f, g), confirming that position of blue modulation as well as location of blue had been learned. These locations were learned relative to a strong green modulation or vertical edge in the apparatus, perhaps at the edges of the targets $[14,15]$.

\section{The bees' world is sketched in green contrast on a panorama in blue}

Relative vertical positions of two large black rectangles on white were easily distinguished (Fig. 7a), although bees have no receptors for white or black. With two targets equiluminant to green (Fig. 7b), the bees detected a difference in positions of blue, which they learned from the white rectangles, and the position of strong green modulation at the vertical edges of buff. With two targets equiluminant to blue receptors (Fig. 7c), they used the only remaining cue, position of strong green modulation (arrows). When a large $\left(20^{\circ}\right.$ diameter) blue spot replaced each black area, the preference reversed (Fig. 7d) because the blue spot displayed more blue than adapted white background, and some white had replaced black. In our own case, so that we can look into dark corners, our eyes adapt rapidly and locally like those of the bee, but we do not see a brightly coloured area when a black spot is unexpectedly removed.

After a short search, spots were found with a shade of green so that the position of blue exactly cancelled the position of green modulation (Fig. 7e). As a result, the trained bees detected the differences in positions of the blue and spots but had no net preference for either target. This result suggests that separate positive (approach) and negative (avoid) signals cancel each other within the memory. 
An apparently simple example is unexpectedly complicated

The large white panel was not distinguished from the general background, and blue receptors became adapted to it.

In an area where black was removed, the blue content of the newly exposed white background had a strong effect

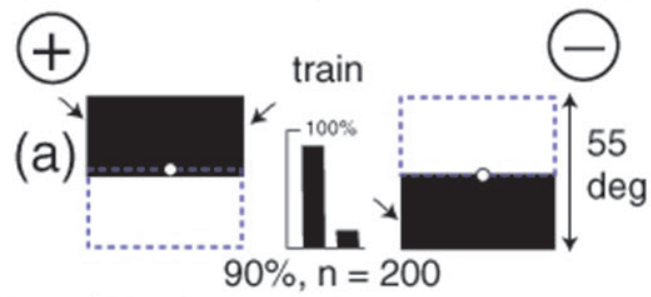

Cues were position of blue in the white, and green modulation (arrows)

(b)

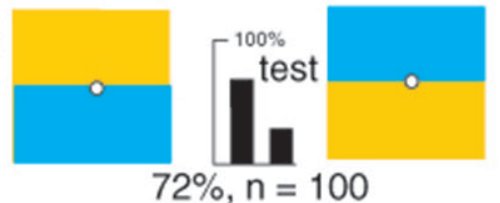

When equiluminant to green receptors, they used the position of blue content

(c)

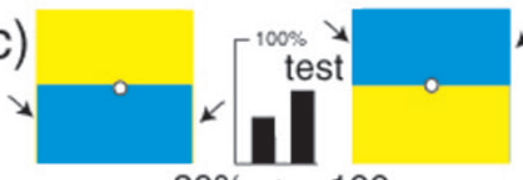

$39 \%, n=100$

When equiluminant to blue receptors, they used the only remaining cue, position of green contrast (d)

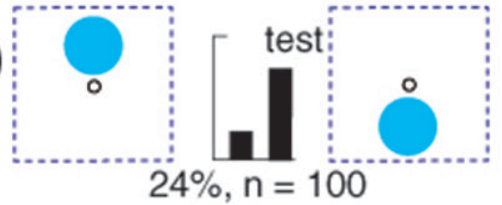

The preference was reversed because the spots appeared more blue than adapted background

These results illustrate the extreme anti-intuitive nature of bee vision data from Horridge 2014

$(\mathrm{e})$

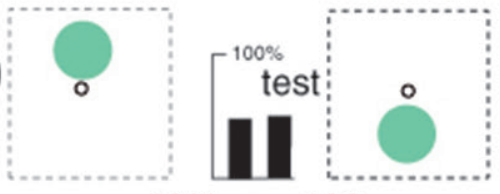

$49 \%, n=100$

The green spots were equally attractive because the position of blue in the displayed white just balanced position of green contrast

Fig. 7. Blue receptors adapt to blue content of white background. (a) Bees were trained to locate black as absence of blue (in the white). (b) With patterns of buff and blue, equiluminant to green, they preferred blue at the bottom. (c) When equiluminant to blue they preferred the strongest edge modulation at the top, as in the training. (d) With blue spots, the preference was reversed because blue spots are bluer than adapted background white. (e) A spot colour can be found such that the position of blue (in the white) cancelled the position of green contrast on each target, so the preference was lost 
Patterns of yellow on black effectively restrict the bees to learning the position and measure of green modulation

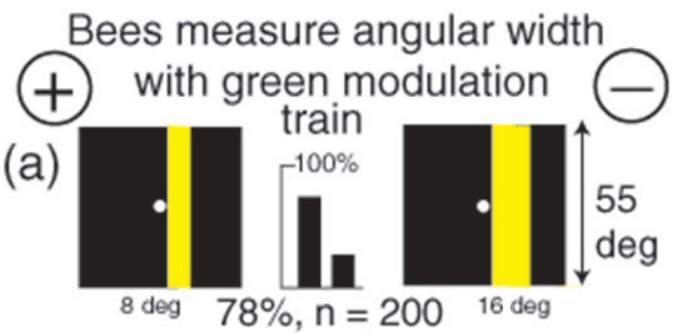

With little blue content and equal modulation the only cue was the width of the bars

(b)

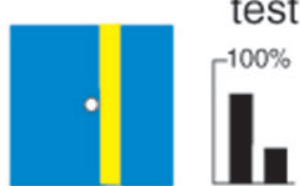

$70 \%, \mathrm{n}=200$

With no blue difference, green contrast showed the edges

(C)

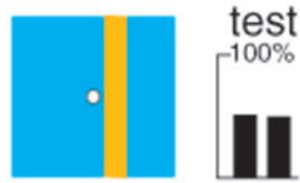

$51 \%, n=200$

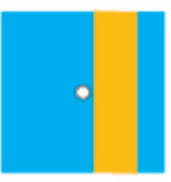

Useless with no green contrast

(f)
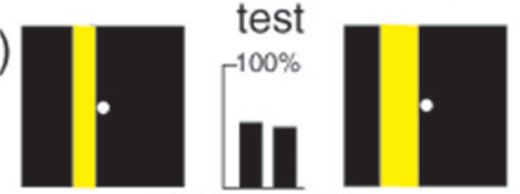

$53 \%, n=200$

Bars were distinguished only in the training position

Fig. 8. Bees learned bar width when there was no other cue. (a) Training patterns of hemp bars on black displayed equal amounts of vertical edge and negligible blue difference. ( $b$ and $c$ ) The bees required green contrast (hemp on ultramarine), not blue contrast (buff on blue) to discriminate. (d and e) Trained bees detected the difference in width of white bars. (f) Memory was retinotopic 


\section{Response to background adapts, but black in memory remains dark-adapted}

This brings us to our fourth awkward informative fact about insect vision, that removal of black or yellow exposes background that has not been locally adapted away or averaged in large areas of green or white $[5,13,14]$. Bee receptors lose nine tenths of their sensitivity in $1 \mathrm{sec}$ when suddenly illuminated $[2,17]$ and the behavioural response is faster still. In the past, all experimenters have used patterns on a white or black background, both of which introduced into experimental procedure inputs that were never suspected. White to the bees looks bluer than blue (Table 1). Black or yellow generated no cue except edge and width, but surprise, surprise, when removed, bees make unexpected choices because they detect strong blue (Fig. 6e) at that retinotopic location where white is exposed and their memory is dark-adapted $[5,13,14,16]$.

\section{With no other cue available, bees measure angular width between vertical edges}

A small group of bees was trained to distinguish a narrow yellow vertical bar on a black background from a similar bar twice as wide (Fig. 8a). These patterns had little blue content and equal modulation at the edges of the bars, leaving little for the bees to learn. They easily distinguished similar targets equiluminant to blue receptors (Fig. $8 b$ ) showing that they had relied on green contrast despite the fact there was no difference in green modulation, but failed with similar targets with no green contrast (Fig. 8c). They had compared the widths of the vertical bars, as shown by tests with thin white lines or bars that displayed green contrast at the same widths between edges (Fig. 8d, e). When the training bars were shifted sideways on the targets, the bees failed because recognition was restricted to the place on the eye where learning had occurred (Fig. 8f). This usually happens with fixed (not shuffled) targets.

\section{Bees do not recognize shape, but they distinguish shapes by other cues}

A group of bees was trained to discriminate a yellow diamond on a black background from a square of the same shape and size on a similar background (Fig. 9a). This looks like shape recognition [18]. In human vision, it is shape discrimination. In bee vision, it is usually so called because the bees certainly learn to discriminate, but a few tests reveal that they learned to avoid the target that displays the greater green modulation (Fig. 9b-g, k). They were unable to find a cue in gratings equiluminant to green receptors (Fig. 91). The trained bees preferred an unfamiliar version of the shape (Fig. 9g). The trained bees recognized neither of the training shapes. They failed to recognize the rewarded target (Fig. 9h) and the unrewarded one (Fig. 9j). Finally, a critical test revealed the cue. The trained bees avoided the greater green 


\section{Train, with green contrast}

(a) but little blue content

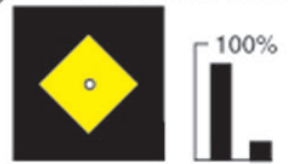

$84.0 \%, n=200$

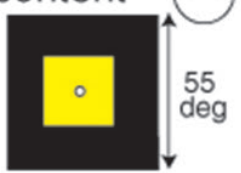

With negligible blue and equal areas of yellow,

the only available cue was green modulation

(b)

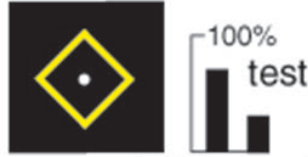

$73 \%, n=100$

(c)

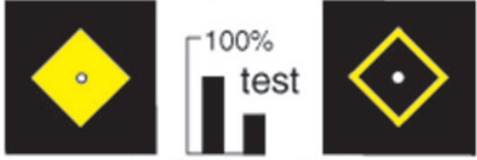

$67 \%, n=100$

(d)

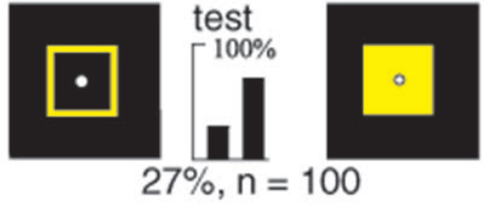

(e)

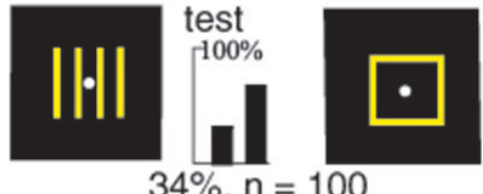

(g)

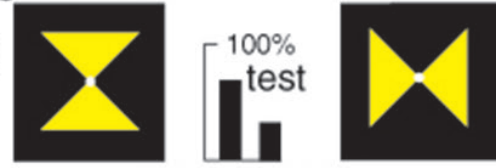

$69 \%, n=100$

(h)
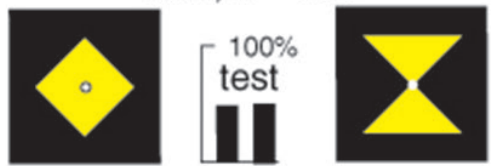

(j)

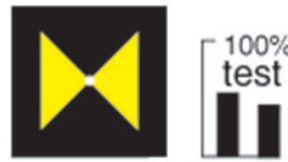

$55 \%, n=100$

(k)

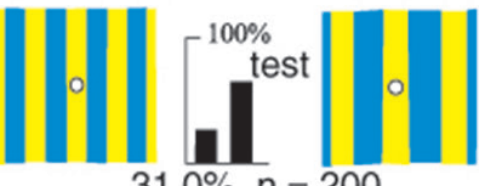

$31.0 \%, n=200$

In all of the above, bees avoided greater green modulation at vertical edges, irrespective of shape. In (d), (e), (h) and (j) the square was not recognized.

(f)

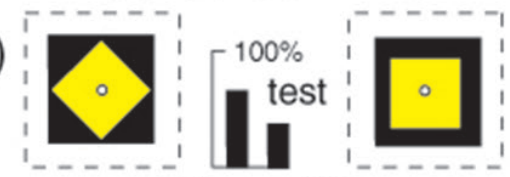

$65 \%, n=100$

Replacing some black with blue reduced the score
(I)

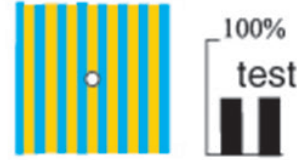

$49.5 \%, n=200$

With no green contrast there was no cue

Fig. 9. Bees use modulation to distinguish shapes. (a) Training patterns. (b to g, and k) Trained bees avoided targets with most vertical edge irrespective of shape. (h and $\mathrm{j}$ ) They fail to recognize the training shapes. (1) They were lost without green modulation 
When trained with one colour versus another, the preferred cues were a measure of blue content and location of the strongest green modulation

Trained with equal green contrast at †vertical edges, they avoided blue

(a)

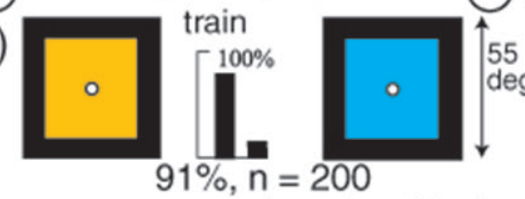

Trained with small blue difference, they avoided

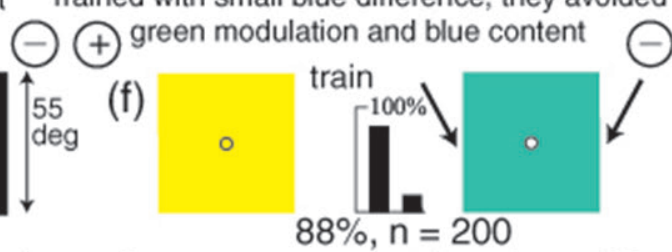

Contrasts measured against black

Contrasts measured against white

(b)

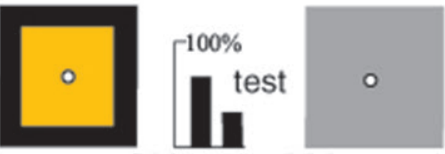

$69 \%, n=200$

Trained bees avoided the greater blue content

(c)

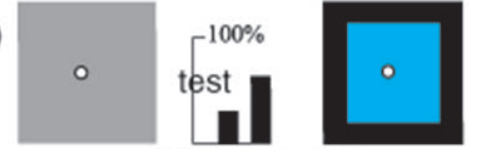

$35 \%, n=100$

Trained bees avoided the greater blue content

(d)

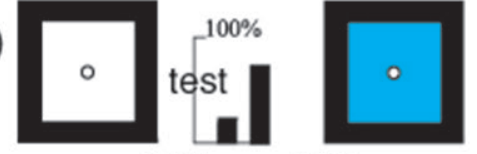

$26 \%, n=200$

Trained bees avoided the greater blue content

(e)

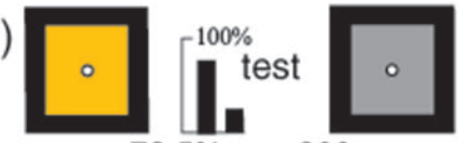

$78.5 \%, \mathrm{n}=200$

Trained bees avoided the greater blue content (g)

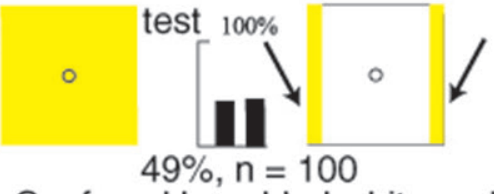

Confused by added white and similar green contrast at edges

(h)

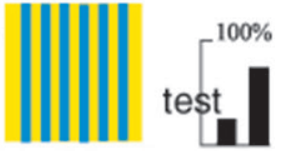

$25 \%, n=100$

They avoided greater green modulation in gratings equiluminant to blue

(j)

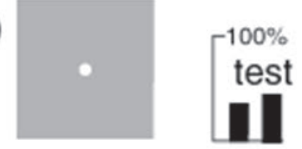

$40 \%, n=100$

Similar green contrast but avoided greater blue content

(k)

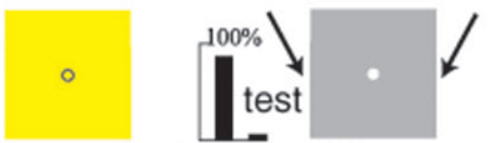

$96 \%, n=200$

Better than training; avoids greater blue content and also green contrast,

Fig. 10. Colours are distinguished by blue content and green modulation. (a and f) Training patterns of buff and blue with no green difference. (f) Of ultramarine and hemp with no blue difference. (b to e) Trained bees avoided the greater blue content irrespective of the test patterns. $(\mathrm{g})$ Bees failed when tested with similar edges. (h to $\mathrm{k}$ ) They had learned to avoid green edge modulation (arrows) 
modulation in gratings equiluminant to blue receptors and equal in average colour (Fig. 9k).

This example illustrates how logical deduction of inputs from numerous varied test results replaces speculation based on training only. With two different shapes of equal area of the same colour, a measure of blue content is useless, and if available, a measure of modulation difference is the most preferred cue (Figs 3, 10f-k), before measurement of widths (Fig. 8). Therefore, discrimination of equal areas of the same colour on a black background (Fig. 9a) must be poorer as less green contrast is available when their training colour is changed from yellow towards blue. Bees trained to distinguish a square and a diamond of various colours, with no tests of what they had actually learned, indeed scored highest when yellow and lowest when blue [19] but it was an error to conclude that they distinguished shape.

\section{Discrimination of colour depends on blue content and green modulation}

Two examples given here illustrate the extremes of colour discrimination. In the first, two targets of buff and blue were equiluminant to the green receptors but a group of bees learned quickly and well (Fig. 10a). When each training target was tested versus a plain grey one ( $40 \%$ white) the trained bees preferred the target displaying buff but avoided the one displaying blue (Fig. 10b, c) because in each case they had been trained to avoid the greater blue content (Table 1). The awkward informative fact is that they avoided white and abandoned the blue (Fig. 10d) and preferred buff to grey (Fig. 10e). In all these tests, they merely avoided the greater blue content. This is exactly what puzzled von Hess so long ago, when he trained bees to go to yellow. They learned by trial and error to avoid blue and would accept any other colour [7]. Von Hess had the crucial data but did not understand his own results.

In the second example, two targets of ultramarine and hemp were equiluminant to the blue receptors and a group of bees learned more slowly (Fig. 10f). A test with the rewarded hemp colour versus a target with hemp edges failed (Fig. 10g) and they avoided the greater green modulation when tested with gratings with green contrast but no blue contrast (Fig. 10h). They found grey and green rather similar (Fig. 10j), and preferred plain hemp because it displayed very little green contrast against white background (Fig. 10k). There was no blue difference in the training, so green modulation at vertical edges had to be the cue, and test colours were irrelevant.

\section{Green, white and grey were distinguished like any other colour}

Green was confused with shades of grey or yellow in the early experiments of von Frisch $[4,14]$. In animals with trichromatic or dichromatic colour vision, a mixture of blue and yellow can be found that matches any green. Bees are not particularly 
Green, greys and white were distinguished like any other colour † Train versus various greys $\odot \oplus$ Train on green vs white -

(a)
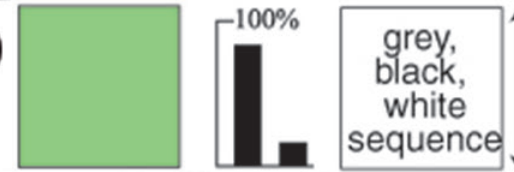

$89.5 \%, n=200$

Greys and white were averaged

(b)

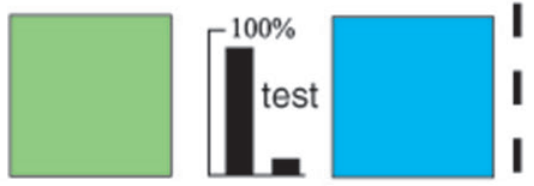

(c)

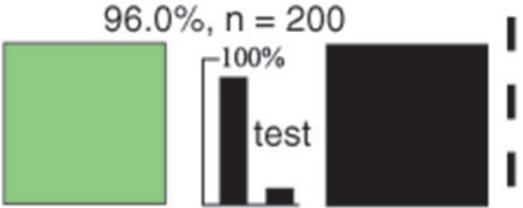

$97.5 \%, n=200$

(d)

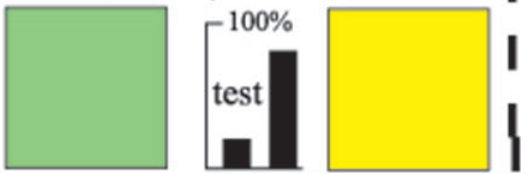

$16.5 \%, n=200$

Avoided blue and green modulation I

\section{(g) 55 deg}
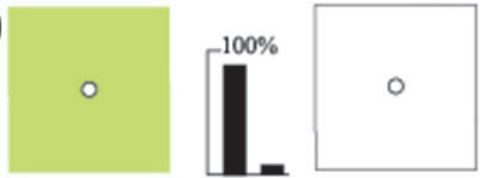

$94.0 \%, n=200$ after $2 h$

(h)

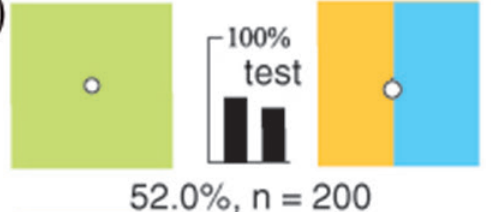

(j)

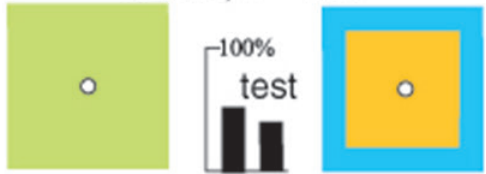

$55.0 \%, n=200$

Blue plus buff were summed

(k)

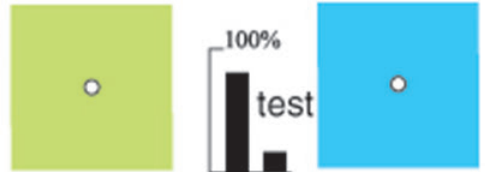

$89.0 \%, n=200$

(l)

(e)

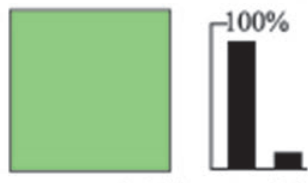

$91 \%, n=200$

(f)

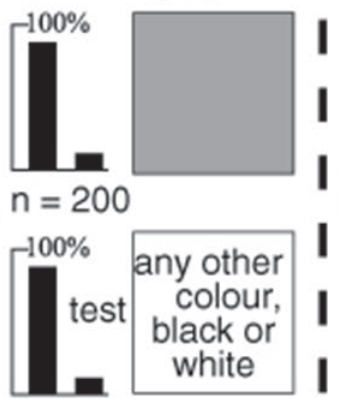

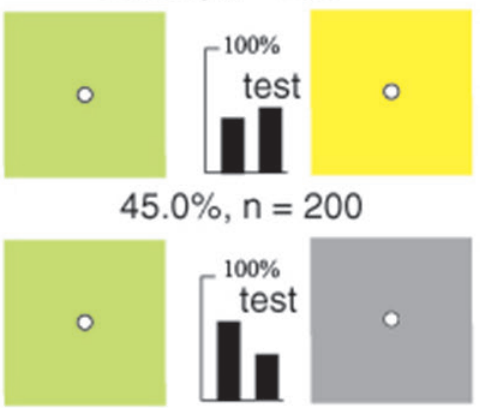

$64.5 \%, n=200$

They avoided the most blue

Always preferred yellow; to avoid blue

Fig. 11. Green was distinguished like any other colour. (a) Training patterns. (b-d) The trained bees avoided blue, and green contrast. (e, f) When trained on green versus grey, they avoided blue content in any other colour. (g-m) After training on green versus white, they avoided the most blue. (h and j) Buff and blue were added tyo make green. Green contrast was similar at outer edges 


\section{Just a few of the infinite number of pairs of patterns that are not distinguished at a distance}

(a)

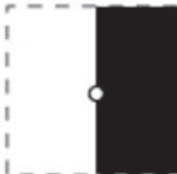

White merges into background. Isolated black is not located

Edges have no polarity or difference. score $58.0 \%, n=200$ after $3 h$

(b)
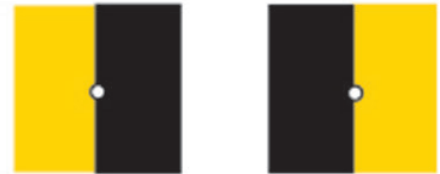

No colour difference or central polarity. Green contrast at edges was saturated Targets were effectively black all over score $52.5 \%, n=200$

(c)
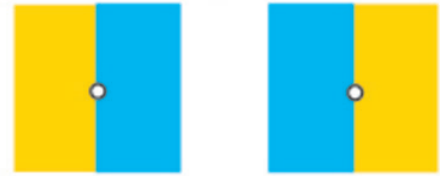

Equal summed colour content Equiluminant to green receptors. Outer edges equal green contrast. score $51.7 \%, n=300$

(d)
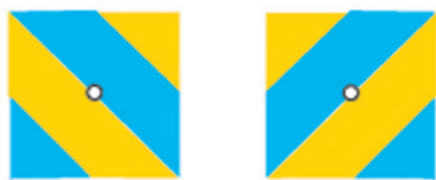

Equal summed colour content

Equiluminant to green receptors Blue contrast conveys no orientation score $47.7 \%, n=300$
55 (e)
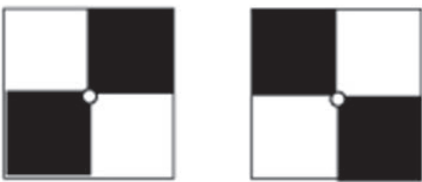

White merges into background Black not located horizontally and total black equal vertically score $50 \%, n=200$

(f)
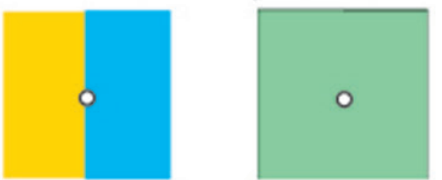

Equal summed colour content Equiluminant to green receptors. Outer edges equal green contrast. score $49 \%, n=200$

(g)
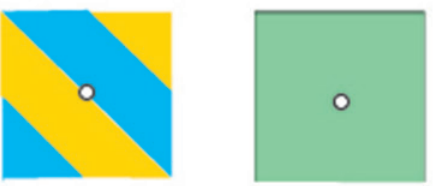

Equal summed colour content Equiluminant to green receptors. Outer edges equal green contrast. score $48 \%, n=100$

(h)
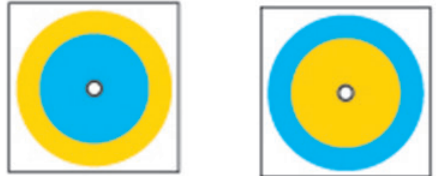

Equal summed colour content Equiluminant to green receptors Blue contrast conveys no orientation score $53 \%, n=100$

For every shade of colour, there is a another colour equiluminant to the green receptors and another equiluminant to the blue channel

Fig. 12. Some of the numerous pairs of patterns that bees do not distinguish 
attracted to green, presumably because they seek nectar and pollen, not foliage, and for a long time mystery has dogged the bees' memory of green and white $[3,4,9,20]$.

When bees were trained to green versus a random sequence of black, white and grey levels (Fig. 11a), they avoided the greater blue content (Fig. 11b, d) or the greater green modulation at the edges (Fig. 11c). That is what they had available in experiments of von Frisch [4]. When trained with green versus grey ( $40 \%$ white), they preferred hemp to any other colour in tests because hemp always displayed less blue content than any other colour (Fig. 11e, f).

Finally, a group of bees was trained with green versus white. It is easy to predict that in every test they showed that they had learned to avoid the most blue content (Fig. 11k, 1, m), and when the blue content was equal, they were lost (Fig. 11h, j). In these experiments, green behaved like any other colour.

\section{There exist pairs of patterns that are not distinguished by bees}

For a variety of reasons, many pattern pairs are confused (Fig. 12). The failure of bees to discriminate the polarity of a black/white boundary has been hidden in the literature for 85 years [5]. With equal blue content, differences can disappear when contrast becomes saturated at outer edges of targets (Fig. 12b). A combination of no green contrast, equal average colour, and all outer edges similar in contrast (Fig. 12c, d), is not discriminated from its mirror image $[5,11]$. Colour was always averaged over the whole target unless there was a boundary displaying green contrast (Fig. $11 \mathrm{~h}, \mathrm{j}$ and Fig. 12c, d, h).

These pairs of patterns are further indications that bees fail to identify quite large areas of different colours and do not re-assemble coloured patterns [13-16].

\section{DISCUSSION}

\section{Three independent variables, together with angles between them, carry the input}

Bees always scan as they fly, so generating the modulation that is essential for their vision. It is an observable fact that bees do not notice the sign of the change in modulated input nor the polarity at a boundary, so the detectors must be symmetrical about a vertical axis (at right angles to the direction of scanning). It is a simple fact that the steady state value of a variable is independent of the rate of change of the variable. Because each colour approximates to a steady-state and modulation resembles a rate of change, it is convenient to use ideas from the Calculus in a diagram of a minimum model of the conclusions (Fig. 13).

The analysis proceeded on the assumption that the bees failed when they did not detect a cue, and the inputs must resemble those seen in electrophysiological experi- 


\section{Three input channels in parallel}

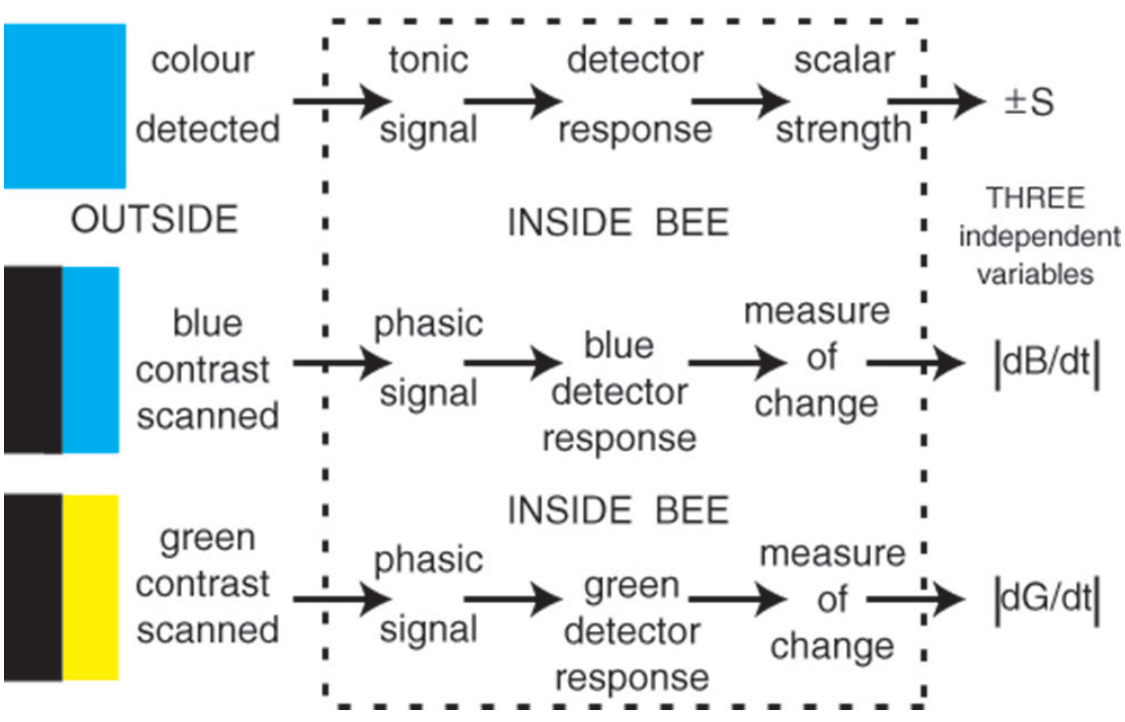

Fig. 13. Three separate and unexpected input channels in parallel delayed the analysis of the mechanism and obscured the shifts in preference as different patterns were trained and tested

ments. The ideal analysis is to search for the minimum number of independent variables logically derived from the data, not to invent causal factors that would be consistent with the data. Our final awkward informative fact is that human intuitive invention of causal factors was usually wrong.

\section{Correlation with neuron arrangement in retina, lamina and medulla}

There is considerable agreement between the inputs deduced behaviourally and some identified neurons. Three types of receptors is a good starting point, although their distribution is not absolutely regular in the bee. There are six green, one blue and one UV receptor in each ommatidium [21]. The green receptor axons end at the lamina. Lamina neurons are better identified in the fly. The large ones on the green pathway are phasic, that is, adapt rapidly and respond to the first derivative of the signal. Blue and UV receptor axons pass through to the medulla (Fig. 14) where UV and green inputs inhibit neurons on the blue pathway. In Drosophila, groups of about 12 blue receptor axons terminate directly on horizontal dendrites of Tm9 neurons, as if summed in groups, which fits the local or total summation of blue content. Probably all hemi-metabolous insects are similar. 


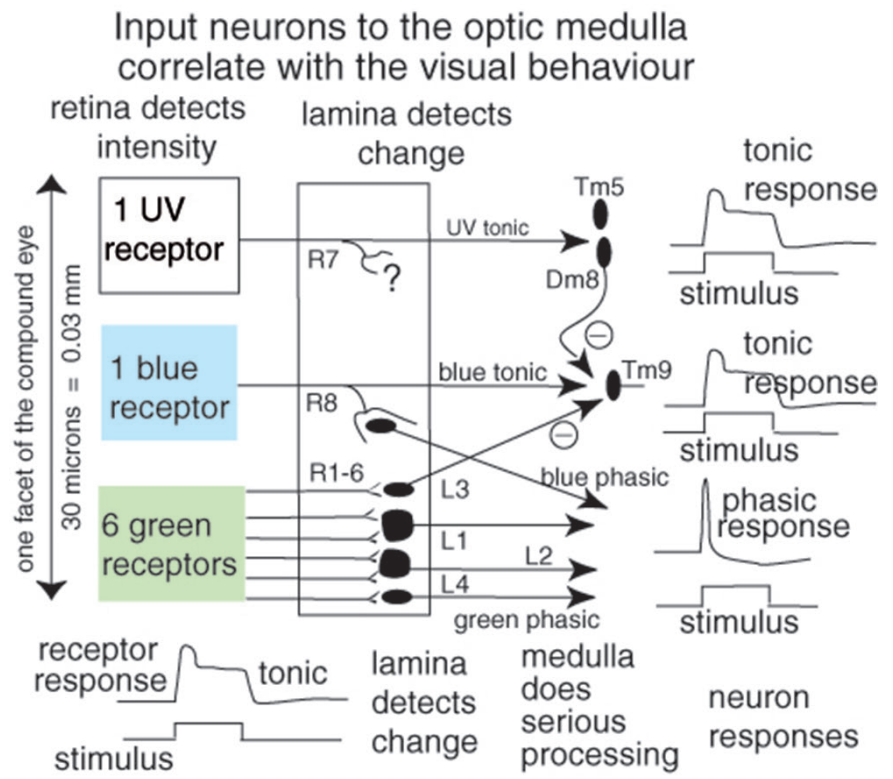

Fig. 14. Neurons that project within a single column below an ommatidium. Six green receptor axons end at the lamina. One blue and one blue receptor axon run into the medulla. UV and green inputs inhibit the blue channel. Corresponding neuron identities were borrowed from work on Drosophila

\section{Detection of modulation and blue content in areas combines structure with colour}

Bees do not detect colour alone; they detect an inseparable mixture of adapted blue content and spatial derivatives of green and blue displays, together with angular separation of edges. All they need is a sparse sampling of cues and angles between cues to identify a place where they previously found food. They are not interested in flowers, pattern, texture, shape, symmetry or colour in the abstract.

Hertz [8-10] had agonized for years in her brilliant search for the minimum inputs in bee colour vision, but knew nothing about numbers and properties of receptor types. In her final paper, although she considered the trichromatic theory [10], she was well aware that figural intensity as a variable was distinct from memory of colour and location [22]. Her tests showed that the trichromatic colour theory could not account for bee colour vision because UV inhibited white (blue) inputs [9, 10]. Until 1999 [11], that was the last effort of the pre-war research tradition on bee colour vision.

\section{Standing on shoulders, or shooting them down}

The early giants knew nothing about numbers or spectral sensitivity of the receptors, so they could not use pairs of equiluminant patterns. An alternative to human-like 
colour vision for bees did not appear until the end of the twentieth century. Progress was probably slowed because von Frisch and his legacy took a stand in defiance of von Hess. It was disgraceful that neither they nor their pupils repeated the experiments of the other. The simple conclusion that bees detected yellow like black would have solved half the problem, but it is now clear why neither understood their own data, and there was no other available theory. Later work was about performance, not mechanism, and failed to follow developments in neuron physiology or artificial vision. So, presumably many anomalous observations were never published. The question, now we have a new theory, is how to re-interpret, not throw away, the collected data of a century on bee colour vision, and to make quantitative studies of the new variables.

\section{Seeing things is not simple, and best avoided}

Bees illustrate very well the problems of trying or evolving to see any thing or shape [19]. At the receptors, the panorama is divided, and captured by every receptor separately so the image has to be re-assembled. Bees have too few output neurons to memory to do that, so they are obliged to detect a few simple cues. Next, the image moves across the eye and detects edges and areas that behave differently. Edges are thin and require small fields to be located; areas require larger fields. The eye must move in order to see, but that smears the image across the receptor fields, which destroys edges and spoils the location of areas. Bees cannot measure contrast, only modulation (Fig. 3), so they cannot separate colour from pattern. Besides, they throw away the polarity of contrast (Fig. 2) and detect the location of maximum modulation [16], which is a product of contrast and length, neither of which can be recovered separately from the display, so pattern, colour and shape are lost. Blue content and edge orientation are each summed over local areas, and the original responses of individual green feature detectors all lost. The essential processes that reduce the information load prevent the bees seeing like humans. However, they are better than an expensive camera, which detects and remembers much but sees nothing.

\section{Bee vision is anti-intuitive for humans but not for robots}

When I look at something, I cannot prevent myself from seeing shapes, colours and textures. However, there are other ways to approach the design of visual systems for robots. It is frankly unwise to start by generating something like human vision, with camera pixels, a raster in Cartesian co-ordinates, efforts to detect shapes from closed contours, and categorize objects in the way that humans cannot avoid. Available features in the panorama are too numerous and too diverse to be absorbed, and the essential combinations that enable understanding or even recognition are very large with a stationary image; never mind a moving one. That way, the combinatorial explosion rapidly becomes an insoluble problem that can be overcome only by a huge computer comparable with the human brain. 
Bee vision solved this problem and served insects well for about 400 million years. We should not blindly copy bees, however, because their vision is adapted to the rapid recognition of place by the coincidence of two or three simple cues sufficient for a herbivore. Bees do not see things; they recognize places where they have learned the location of food and recently revisited. The inputs are incredibly simple, the sampling sparse, and the memory very limited. Similarly, every artificial seeing system can be task-oriented to simplify its design.

\section{REFERENCES}

1. Autrum, H., von Swehl, V. (1962) Die Spektralempfindlichkeit einzelner Sehzellen des Bienenauges. Zeitsch. vergl. Physiol. 48, 357-384.

2. Baumann, F. (1975) Electrophysiological properties of the honey bee retina. In: Horridge, G. A. (ed.) The compound eye and vision of insects. Clarendon Press, Oxford. pp. 53-74.

3. Chittka, L. (1999) Bees, white flowers, and the colour hexagon: a reassessment? No, not yet. Naturwissenschaften 86, 595-597.

4. Frisch, K. von (1914) Der Farbensinn und Formensinn der Bienen. Zool. Jahrb. Physiol. 35, 1-188.

5. Friedlaender, M. (1931) Zur Bedeutung des Fluglochs im optischen Feld der Biene bei senkrechter Dressuranordnung. Zeitsch. vergl. Physiol. 15, 193-260.

6. Hess, C. von (1912) Vergleichende Physiologie des Gesichtssinnes, Gustav Fischer Verlag, Jena.

7. Hess, C. von (1918) Beiträge zur Frage nach einen Farbensinne bei Bienen. Arch. für die gesamtpe Physiol. 170, 337-366.

8. Hertz, M. (1933) Über figurale Intensität und Qualitäten in der optische Wahrnehmung der Biene. Biologische Zentralblatte 53, 10-40.

9. Hertz, M. (1936) Beitrag zum Farbensinn und Formensinn der Biene. Zeitsch. vergl. Physiol. 24, 413-421.

10. Hertz, M. (1939) New experiments on colour vision in bees. J. Exp. Biol. 116, 1-8.

11. Horridge, G. A. (1999) Pattern vision of the honeybee (Apis mellifera). The effect of pattern on the discrimination of location. J. Comp. Physiol. A 185, 105-113.

12. Horridge, A. (2012) The anti-intuitive visual system of the honeybee. Acta Biol. Hung. 63 (Suppl. 2). $146-161$.

13. Horridge, A. (2014) How bees distinguish black from white. Eye and Brain 6, 9-17.

14. Horridge, A. (2015) How bees discriminate a pattern of two colors from its mirror image. PloS ONE $10,1-23$.

15. Horridge, A. (2015) How bees distinguish colors. Eye and Brain 7, 17-34.

16. Horridge, A. (2015) How bees distinguish patterns by green and blue modulation. Eye and Brain 7 , 83-107.

17. Laughlin, S. B., Hardie, R. C. (1978) Common strategies for light adaptation in the peripheral visual systems of fly and dragonfly. J. Comp. Physiol. 128, 319-340.

18. Marr, D. (1982) Vision. Freeman, San Francisco.

19. Morawetz, L., Svoboda, A., Spaethe, J., Dyer, A. D. (2013) Blue colour preference in honeybee distracts visual attention for learning closed shapes. J. Comp. Physiol. A 199, 817-827.

20. Vorobyev, M., Hempel de Ibarra, N., Brandt, R., Giurfa, M. (1999) Do "white" and "green" look the same to a bee? Naturwissenschaften 86, 592-594.

21. Wakakuwa, M., Kurasawa, M., Giufa, M., Arikawa, K. (2006) Spectral heterogeneity of honeybee ommatidia. Naturwissenschaften 92, 464-467.

22. Zerrahn, G. (1933) Formdressur und Formunterscheidung bei der Honigbiene. Zeitsch. vergl. Physiol. $20,117-150$ 